\title{
User-centred design and evaluation of a tele-operated echocardiography robot
}

\author{
Manuel Giuliani $^{1}$ (D) - Dorota Szczęśniak-Stańczyk ${ }^{2} \cdot$ Nicole Mirnig $^{3} \cdot$ Gerald Stollnberger $^{3} \cdot$ Malgorzata Szyszko $^{4}$. \\ Bartlomiej Stańczyk ${ }^{4} \cdot$ Manfred Tscheligi $^{3}$
}

Received: 22 August 2019 / Accepted: 25 November 2019 / Published online: 16 March 2019

(C) The Author(s) 2020

\begin{abstract}
We present the collected findings of a user-centred approach for developing a tele-operated robot for remote echocardiography examinations. During the three-year development of the robot, we involved users in all development stages of the robot, to increase the usability of the system for the doctors. For requirement compilation, we conducted a literature review, observed two traditional examinations, arranged focus groups with doctors and patients, and conducted two online surveys. During the development of the robot, we regularly involved doctors in usability tests to receive feedback from them on the user interface for the robot and on the robot's hardware. For evaluation of the robot, we conducted two eye tracking studies. In the first study, doctors executed a traditional echocardiography examination. In the second study, the doctors conducted a remote examination with our robot. The results of the studies show that all doctors were able to successfully complete a correct ultrasonography examination with the tele-operated robot. In comparison to a traditional examination, the doctors on average only need a short amount of additional time to successfully examine a patient when using our remote echocardiography robot. The results also show that the doctors fixate considerably more often, but with shorter fixation times, on the USG screen in the traditional examination compared to the remote examination. We found further that some of the user-centred design methods we applied had to be adjusted to the clinical context and the hectic schedule of the doctors. Overall, our experience and results suggest that the usage of user-centred design methodology is well suited for developing medical robots and leads to a usable product that meets the end users' needs.
\end{abstract}

Keywords User-centred design $\cdot$ Tele-operated robots $\cdot$ Echocardiography $\cdot$ Medical robotics

\section{Introduction}

The World Health Organisation (WHO) regularly assesses the number of people working in the healthcare sector (doctors, nurses, other healthcare personnel) for all countries that publish numbers about employment in the healthcare sector. In the latest employment statistics, the WHO reports that in 2016 there was an estimated global needs-based shortage of health care workers of about 17.4 million workers [33]. The shortage of workers in the health care sector is also addressed in the WHO's strategy for human resources for health which contains a global milestone for 2030 that "all countries are making progress towards halving inequalities

Manuel Giuliani

manuel.giuliani@brl.ac.uk

Extended author information available on the last page of the article. in access to a health worker." World Health Organization [30]. The usage of tele-operated robots for remote examinations is one way to guarantee better access to health specialists for people living in underdeveloped and rural areas. For example, Boman et al. [8] showed that the use of robot-assisted remote echocardiographic examination drastically reduces the time to diagnosis in rural areas. In a clinical trial that took place in the north of Sweden, they randomly assigned 19 patients to remote consultation and imaging, and 19 to standard consultation. The results of the study showed that the total process time from when the patient first contacted the doctor to when a correct diagnosis was issued was significantly reduced from 114 days to 26.5 days when using remote examination for consultation. The process time was reduced so drastically, because in a traditional consultation, patients first report to their primary healthcare centre and then, based on their condition, have to travel to secondary/tertiary centres for further examinations. When using remote examinations, patients only have 
Fig. 1 The ReMeDi system enables a doctor (left) to execute an echocardiography examination with a remotely controlled robot (right)

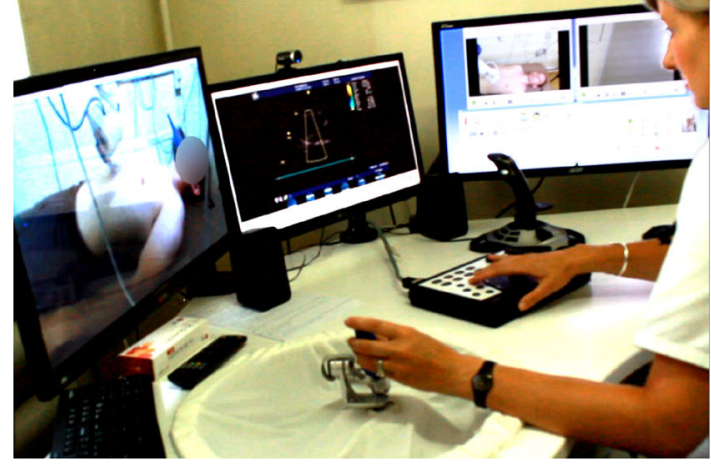

(a) Doctor with remote control interface.

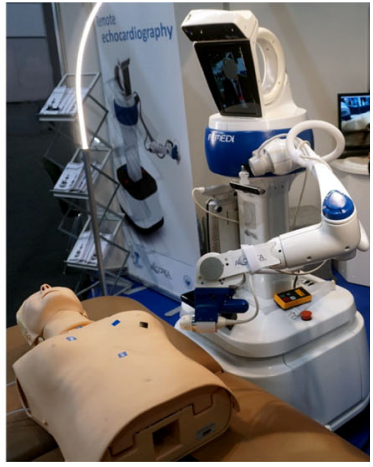

(b) Robot and patient to travel to their primary healthcare centre, but still can get access to additional expertise from doctors, who execute examinations remotely from other centres.

In the ReMeDi project (Remote Medical Diagnosti$\operatorname{cian}^{1}$ ), we developed a robot system for remote echocardiography (Fig. 1). Shaw showed that the involvement of end users in the development is one necessary part for creating a successful medical product [35]. Thus, we followed a usercentred design (UCD) process for developing the robot. We involved the future end users of the robot (i.e., echocardiography specialists) in all development phases: requirement analysis, iterative system development, and system evaluation. In this paper, we report the collected findings of working together with end users to develop the ReMeDi robot. The main contributions of this work are: (1) Results of two eye tracking studies to compare traditional echocardiography examinations to remote examinations with the robot (Section 6); (2) results and best practices from the iterative development sessions with medical end users (Section 5); (3) a report on UCD methods to involve medical end users in requirement analysis (Section 4); and (4) an extensive discussion of the practicability and limitations of the UCD approach for developing a medical robot in an everyday clinical environment and the usability of eye tracking as comparative tool between traditional and remote examinations (Section 7). The main aims of this work is to provide new insights in using eye tracking as tool to compare task efficiency in traditional and tele-operated medical examinations and to share best practices when applying UCD methods in clinical practice.

\section{Related work}

Robots have been used for medical applications since the 1980s. The first medical robots were parts of computer-integrated surgery systems, e.g., [19]. Taylor and Stoianovici [40] give an overview for the first two decades

\footnotetext{
${ }^{1}$ http://www.remedi-project.eu
}

of research on surgery robotics. Since the late 1990s, robots have also been used for ultrasound applications. Priester et al. [31] provide a review of medical robotic ultrasound systems. They define three classes of applications for ultrasound robots: extracorporeal imaging, needle-guidance, and intraoperative surgery. The ReMeDi system that we are describing in this paper (Section 3) is used for extracorporeal imaging, specifically for echocardiography. Other extracorporeal imaging systems have been proposed for ultrasonography (USG) of the abdomen (e.g., [15]), the carotid artery (e.g.,[32]), the lower limbs (e.g., [17]), and for general usage (e.g., [26]). Other groups have proposed systems for remote echocardiography, which are similar to the ReMeDi system. Boman et al. [9] presented the CARDISTA system (CARdiological consultation at a DISTance) and showed that long distance real-time echocardiography is technically possible. Koizumi et al. [18] presented a remote ultrasound diagnostic system for dialysis-related amyloid arthropathy and demonstrated in a diagnostic experiment a successful examination by a doctor on a real patient. Mathiassen et al. [27] showed that it is possible to realise tele-operated ultrasonography with a UR5 robot arm by Universal Robots. The robots of the other groups use similar technology to our system. However, the other groups do not report insights of working together with end users for system development.

We used eye tracking to measure the differences for doctors when conducting a traditional compared to a remote examination. Eye movements can provide insight into cognitive processes, such as attention and mental and visual workload. Eye tracking has been used in various fields, although not in the medical robotics context. The most commonly used eye tracking measurements are glances, fixations, and saccades. According to DIN EN ISO 150071 2013, a glance is defined as maintaining of visual gaze within an area of interest, bounded by the perimeter of the area of interest. A gaze may be comprised of more than one fixation and saccades to and from it. Its duration is measured as glance duration. A fixation is an alignment of the eyes so that the image of the fixated area of interest falls 
on the fovea (the middle of the retina responsible for our central, sharpest vision) for a given time period. A saccade is a brief, fast movement of the eyes that changes the point of fixation. In our eye tracking studies, we measured glances and fixations. Glances are used to measure the general attention of a person towards and area of interest. Fixations are used for visual information extraction, which is relevant for our study, because study from which areas of interest the doctors extract the most information. Saccades are informative for analysis of reading tasks or information search gaze patterns, which are both not relevant in our study.

Comparisons between traditional and remote examinations have been made before, although without using eye tracking. Arbeille et al. [2] asked doctors to execute a teleoperated ultrasound examination on 20 patients. As measurement for the reliability of the remote robotised system, they used the ratio between the number of well-visualised organs with the robotic system compared to the number of visualised organs in the traditional echography. The doctors were able to execute the examination correctly in 18 out of 20 cases, but needed approximately $50 \%$ longer than in a traditional examination. Smith-Guerin et al. [36] validated a tele-echography system on 20 patients. They found that post traumata could be correctly highlighted in $80 \%$ of the cases and four cardiac chambers views had been obtained by the doctor in $90 \%$ of the cases when using the remote system. The doctors were able to detect all digestive systems on liver, gall bladder, pancreas and kidney and all urinary symptoms on bladder, kidney, prostate, uterus and ovaries had been seen with remote and traditional examinations. Martinelli et al. [24] used a robot-based tele-echography system for remote ultrasound examination of the abdomen to examine 58 patients. They compared the diagnostic findings of the remote examination with that of a traditional examination. The doctors were able to find all aneurysms the patients had, using both examination techniques. Georgescu et al. [13] studied a doctor using a tele-operated echography robot for one year in everyday usage. The doctor used the robot 300 times over that year, examining abdominal organs, pelvic organs $(6.7 \%$ of the examinations), supraaortic vessels (46\%), the thyroid $(11 \%)$, on leg veins $(10 \%)$, and on the kidney and urinary tract $(3.7 \%)$. These results show that tele-operated ultrasound is indeed a useful tool for doctors. Adams et al. [1] compared adult abdominal examinations using a teleoperated ultrasound system in a study with 18 patients. The doctors participating in this study identified 5 pathological findings in both examinations, but 3 findings were only identified in the traditional examination, and 2 findings were only identified using the tele-operated ultrasound robot. In all of the above reviewed comparison studies, the authors used either the number of correctly visualised organs or of found traumata as measurement for reliability of the remote examination. In the comparison study reported in this paper, we are focusing on echocardiography, i.e. ultrasonography of the heart. Our measurement criteria for a successful examination is the number of positions on the patient body, from which the doctors were able to correctly visualise the patient's heart.

Finally, we review the application of user-centred design methods in medical robotics. User-centred Design (UCD) is a design methodology that involves the end user of a new product or human-machine interface in all product design stages: analysis, design and development, and evaluation. These stages are usually executed in an iterative, cyclical fashion. Figure 2 shows a general representation of the three stages. UCD has been recognised as product development methodology that leads to a better product usability and higher user acceptance [29]. It has also been formally standardised in ISO standard 9241-210:2019. In the development of the ReMeDi tele-operation system, we involved medical doctors and patients in focus groups and design sessions for analysis of user requirements; during the design phase of the robot we regularly organised feedback sessions with doctors to iterate robot prototypes; for evaluation of the system, we executed comparative eye tracking studies with medical doctors. Throughout this paper, we have used the key words analyis, design, and evaluation in section headers to make it clear to the reader which UCD method we describe in the section.

UCD has been used in the development of other medical technology. Martin et al. [23] summarise user-centred design methods and discuss their applicability for the development of medical devices. They recommend the use of contextual inquiry and ethnography at the beginning of development and other methods, including usability tests and focus groups during developing a new system.

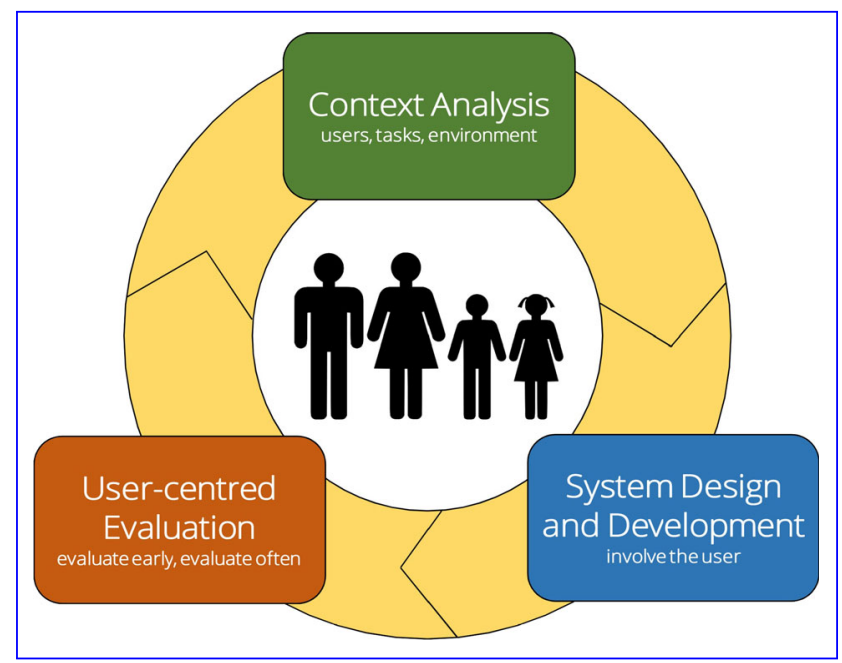

Fig. 2 General overview of a user-centred design process: the user is involved in analysis, design, and evaluation of the new system 
This is similar to our approach for user-centred design of the ReMeDi system. Shah et al. [14] show a survey of literature between 1980 and 2005 in which user-centred design methods have been used mostly for involving users in development and evaluation of healthcare technology. The analysis of the surveyed literature revealed that users are involved to different degrees in the development stages. Most often, they are involved in system design and in taking part in testing and trials. End users are less often involved in the deployment of new healthcare systems and very seldom take part in the concept stages of developing new systems. Similar to our approach, Brandt et al. [10] used a usercentred approach to collect the user requirements for an image-guided orthopaedic surgery robot. They executed a literature review, handed out questionnaires, and conducted workshops with domain experts. Similar to the second part of our requirement analysis, Lee at al. [20] conducted a survey with physiotherapists as part of a user-centred design process. Holt et al. [16] report on the involvement of therapists and users for a robotic system for rehabilitation therapy. They involved users not only in requirement analysis, but also in quarterly reviews to get feedback on system design and development. Lu et al. [22] present a user-centred design process for developing a limb stroke rehabilitation robot, which is similar to our approach. The authors observed stroke therapy sessions and conducted a survey with stroke therapists. They evaluated a first prototype of the robot with stroke therapists in a focus group and a preliminary study.

\section{ReMeDi remote echocardiography robot}

The work carried out and described in this paper has been part of the ReMeDi project. The goal of the project was to develop a robot that enables doctors to remotely execute echocardiography and auscultation on patients. Figure 1 shows the final implementation of the robot. It consists of two parts; one is stationed at the site of the doctor, the other is located at the site of the patient. The doctor controls the robot using a set of input devices (Fig. 1a), including a haptic interface for controlling the robot arm, a dedicated keyboard for the ultrasonic device, and a joystick to adjust the view angle of a camera on the robot to see the patient and robot arm.

The haptic interface was designed to let the doctors perform the same movements as during traditional examinations. The kinematics of the interface [5] cover the required workspace of the doctor and assure the stiffness of the overall device. It provides three degrees of freedom for the translation of the end effector and contains three legs supporting the end effector. Each leg is composed of two rotational joints, four bar linkages and a rotational joint. At the end of the interface, we installed a 3D-printed probe handle that is similar to that of standard ultrasonography probes used for echocardiography.

With a foot pedal (not visible in Fig. 1a), the doctor can switch between two robot arm movement modes. Doctors can either move the arm to position the ultrasonic probe or keep the tool centre point of the arm stable during the ultrasonography examination. The doctor further has three screens that show a live view from the patient site, the image from the ultrasonic device, and a threeway video conference system between doctor, assistant, and patient [39]. The robot is located at the patient site (Fig. 1b). It consists of a mobile platform [4], a screen that shows the doctor's face to the patient, and a light-weight, compliant robot arm that can be equipped with either an ultrasonography probe or a stethoscope end effector. An assistant monitors the examination and ensures the safety of the patient. The two parts of the robot communicate over remote distances via the Internet.

For a general overview of the technical components of the ReMeDi robot please refer to [3]. A technical description of the control architecture is described in [37].

\section{Analysis: user requirements}

As first step in our user-centred design process, we conducted an extensive user requirement analysis. We began with a literature review in the fields of remote ultrasonography, physical examination, and robotic systems within a medical context. In the second step, we executed two observations of a health check performed by a doctor on a patient. The patient received two palpation examinations by the same doctor, the first one during a standard health check, the second one while being ill and having stomach pains. The observations allowed us to get a better feeling for the patients' perspective during a physical examination and to generate a list of typically performed examination steps. In the third step, we organised two workshops with doctors, one in Austria and one in Poland. The main goals of the workshops were to get a deeper understanding for examination techniques, as well as to investigate when and why they are used. We also asked for the doctors' opinions about the usage of robotics systems for remote medical examination. In the fourth step, we organised two focus groups with patients, again, one in Austria and one in Poland. The main goals of these workshops were to get a deeper insight into the patients' perspective concerning examinations and about their opinions and needs regarding examinations executed remotely with a robotic system. Finally, we conducted two online surveys, one with doctors and another one with patients, in order to quantify the insights gained in the workshops. 
The result of these analysis steps was a list of 21 user requirements (11 doctor, 3 assistant, 5 patient, 2 common requirements). In this publication, we focus on the implementation of the requirements by the doctors, hence we only discuss the 11 doctor requirements presented in Table 1. Please refer to [38] for more details of the user requirement analysis.

The first four requirements, RQ1-RQ4, cover aspects of communication and information exchange between doctors, assistants, and patients. In order to meet these requirements, we implemented a three-way video conference system [39] that has separate communication channels between doctor and patient (RQ1), and between doctor and assistant (RQ2). The video conference system also allows submission of additional patient data (RQ3, RQ4). Requirements RQ5 and RQ6 concern the control of the robot arm. For implementation of these requirements, the arm of the ReMeDi robot can be moved by hand, without using an additional steering device (RQ5). We iterated the placement of the camera facing the patient to ensure a visual observation of the patient by the doctor for the entire duration of the examination (RQ6). This is described in more detail in Section 5.2. There were two requirements about the ultrasonography examination, (RQ8, RQ9). We implemented RQ8 in the design of the probe handle used on the haptic interfaces as well as in the kinematics used to control the robot arm with the handle, as described in Section 3. We also implemented the three requirements for the other examination techniques percussion and palpation (RQ7, RQ10, RQ11), but do not discuss these here, since the focus of this paper is on ultrasonography.

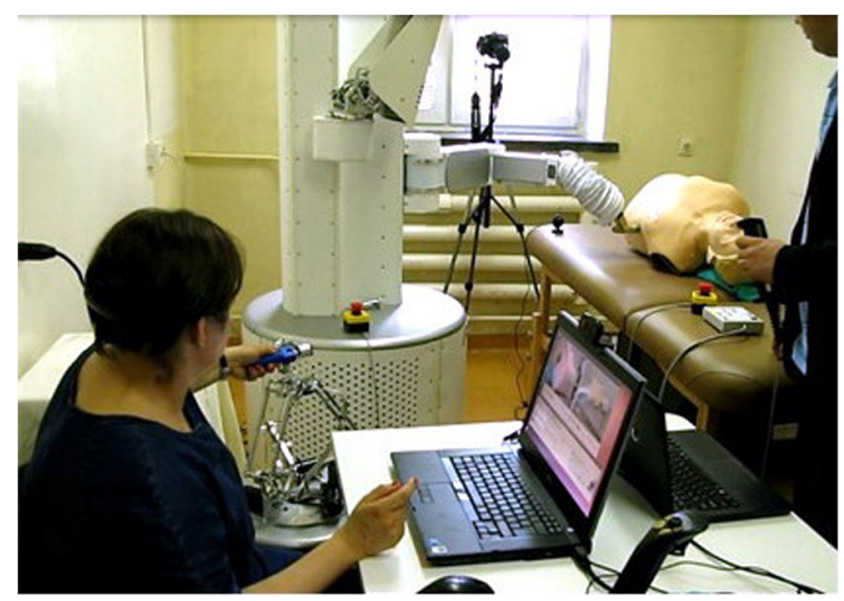

Fig. 3 Usability test with a prototype of the ReMeDi system

We implemented the user requirements in the first prototype of the ReMeDi robot, which can be seen in Fig. 3 . In the following section, we describe how we iterated the design of the robot together with the end users, which ultimately led to the development of the second prototype (Fig. 1) that we then used for the comparative user study described in Section 6.

\section{Design: iterative, user-centred robot development}

In this section, we describe how we involved the end users in the design and development of the ReMeDi system. The

Table 1 User requirements by doctors

RQ1 Doctors want to communicate with the patient during the whole examination using video and audio channels.

RQ2 Doctors want to have an additional communication channel to the assistant that is separated from communication with the patient and can be put into a private mode in which the patient cannot observe the doctor-assistant communication.

RQ3 Doctors would like to observe local changes of the patient's body temperature and assess wetness of patient's skin.

RQ4 Olfactory information can be crucial to make a diagnosis in some cases, for example, if the patient is drunk, has vomited, or has diarrhoea.

RQ5 Regarding the positioning of the robot, doctors want the assistant to position the robot roughly in front of the patient and do the fine-positioning of the system themselves. The doctors have no preference towards a certain input device (e.g., a joystick, 3D mouse, or 3D touch device).

RQ6 For the doctors, it is essential that the robot is positioned in a way that a visual observation of the patient is possible at all times during the examination.

RQ7 In addition to ultrasonography and physical examination, the doctors would also like to perform percussion on the patient. In this examination technique, the doctor taps the thorax or abdomen of the patient to determine the underlying structure.

RQ8 Doctors need to position the ultrasound probe with a vertex angle of at least $60^{\circ}$.

RQ9 Doctors need to see the ultrasound images, preferably in real-time, as the quality of the diagnosis can badly suffer from a long delay in the transmission of the ultrasound images but also in the robot control.

RQ10 Doctors state that it is not really necessary for them to see their own hands when they palpate a patient by the aid of a robotic system. During a traditional examination, they look at the patient's face to monitor their reactions. However, the doctors also mentioned that this could increase the necessary training time, due to their unfamiliarity with robots.

RQ11 It is essential to the doctors that the robotic medical system offers different pressure levels for physical examinations. These should range from very slight, in cases of extreme pain, to hard, which is required for deep palpation. 
goal here is to share our experiences of working together with users from the medical field and summarise the most important findings that are also relevant for development of other medical robots.

During the course of the project, a selected group of five medical doctors from the Medical University of Lublin, Poland, participated in designing and evaluating the ReMeDi system at different development stages. Virzi [42] showed that in a usability test $80 \%$ of the usability problems are detected with four or five participants. The doctors provided their feedback on an ongoing basis as soon as the engineers reached a new stage in the system design process. The group of doctors was involved in the following development steps:

- Preparation of CAD models of the first version of the ReMeDi system. The doctors reviewed and revised the CAD models together with the hardware developers. They systematically evaluated whether the robot would meet the collected user requirements and the medical prerequisites for a successful examination.

- Involvement in evaluation of single robot components. The doctors reviewed several system parts at early development stages: the robot arm, the teleconferencing system [4], and the haptic interface for remote robot control.

- Evaluation of the first version of the integrated ReMeDi system. We report the findings from this evaluation in Section 5.2. The evaluation results directly influenced the design of the second system version.

- Assessment and consultation for the 3D models of the second version of the ReMeDi system. In this step, the doctors were involved in analysing the kinematics of the robotic arm from a medical perspective.

- Evaluation of the second integrated version of the ReMeDi prototype. We report the setup and results of this study in Section 6.2.

In the following sections, we describe details of the evaluation for the first system prototype of the ReMeDi system. This evaluation stands as a good example of how we applied user-centred design methodology to involve the doctors in iterative system development. We review the deployed user-centred design methods in Section 5.1 and report the results of the evaluation in Section 5.2.

\subsection{User-centred design methods}

During the first prototype evaluation, we deployed a set of user-centred design methods. The main user feedback sessions took part in form of scenario-based usability tests. The main goal of these tests was to research whether the doctors consider the remote control of the ultrasonography probe with the ReMeDi system as natural.
We also wanted to know, whether the doctors can reach all necessary positions for echocardiography on a patient's body when using the robot. As safety is of utmost importance in medical devices, we additionally studied whether the doctors consider the tele-operated ultrasonography examination as safe for the patient.

We arranged all usability test sessions in Clinical Hospital No 4 Lublin, which means that we transported and set up the robot on hospital premises for each of the usability tests. Being in the hospital environment increased the authenticity of the tests. Furthermore, it proved to be useful being physically near the doctors. A typical day at the hospital is quite busy and being able to execute usability tests spontaneously to fit into the tight schedule of the doctors led to more opportunities to get valuable user feedback.

The tasks that the doctors had to perform during the usability tests was to execute a remote echocardiography examination on a practice dummy. For this, the doctors had to use the ReMeDi system to remotely place the ultrasonography probe on three pre-defined positions on the body of the dummy. The doctors carried out these tasks under two different conditions. In the first condition, the doctors were able to see the patient practice dummy. This condition was chosen to get a first indication whether the doctors can control the robot at all. Figure 3 shows the setup for this condition. In the second condition, we placed a curtain between doctor and patient, so that the doctors had to use the visual input on the control screens of the system. This condition was chosen to simulate a remote examination, in which the doctor can only rely on the inputs by the robot's sensors. Before executing the tasks, we instructed the doctors how to operate the prototype of the ReMeDi system. The doctors were allowed as much as time as needed to get familiar with the system.

During the usability tests, we used the thinking-aloud method [21].

In this method, the users are asked to actively talk about what they are thinking while they are performing a set of specified tasks with a new user interface. For example, we encouraged the doctors to say what they are looking at, what they think when they look at parts of the system, and how they feel as they go about their task. This enabled the present system developers to see first-hand how real users complete tasks with their system and allowed them to get direct feedback from the users. In addition to the first-hand experience, we also video-recorded all usability tests for further analysis.

\subsection{Robot prototype evaluation results}

A total of 12 doctors (9 cardiologists and 3 radiologists) took part in this usability test. While 5 of the participants 
were our team of associated doctors, the other 7 doctors were staff members of Clinical Hospital No 4 Lublin. We chose to invite additional doctors to use the system to get extra feedback on the robot and to include users in the evaluation, who had not seen the robot before. We sorted the findings of the evaluation into five categories: haptic interface, vision system, interface element layout, robot arm, and general comments.

Haptic interface During the remote examination, a majority of the participants (10 out of 12 doctors) had problems with synchronising the haptic input device to the robot arm. In the first prototype of the ReMeDi system, the doctors had to manually align the rotation and direction of the haptic handle for the ultrasonography probe to that of the robot. The doctors suggested implementing an automatic synchronisation of handle and arm. The doctors furthermore reported a noticeable delay between moving the robot arm with the haptic interface and receiving visual feedback from the robot's camera. They received force feedback over the haptic interface when the probe touched the patient's body, but this feedback was not synchronised with the visual feedback. Six doctors pointed out that it would be useful to have a visual indicator for the forces the robot arm applies to the patient's body. In general, the doctors commented that the haptic interface is working well, but some doctors pointed out that it should have covers to secure the mechanics and motors of the device. The doctors also positively commented that switching between robot movement modes with the foot pedal to either use the haptic interface for moving the arm or for executing the ultrasonography examination works well.

Robot arm All doctors commented that the robot arm in the first prototype of the ReMeDi system was too loud. A loud robot arm could unsettle the patient and lead to difficulties during examination techniques that are sensitive to noise, for example auscultation. The doctors also suggested that the ultrasonic probe always needs to touch the patient's body with its full surface to avoid losing the ultrasonographic image. The doctors found that it would be desirable to separate control of the robot arm rotation from robot arm movement, once the probe is placed into position for examination. Additionally, the doctors reported that the robot's workspace did not cover the entire examination area. The testing with end users also unveiled that it was very easy to drive the robot arm into rotation and translation limits. Finally, the robot sometimes moved rapidly and unpredictably, which could potentially lead to harming the patient.

Vision system In the first prototype of the ReMeDi system, the robot was equipped with two cameras. The first camera was mounted on top of the robot, combined with a screen that showed the doctor's face to the patient. The other camera was directly mounted on the examination settee. The doctors reported that this setup was not usable for remote ultrasonography, because they were not able to clearly see the ultrasonic probe. The robot arm was sometimes blocking the view on both cameras. The doctors suggested to redesign the placement of the camera, to add more cameras to the system, to increase camera shutter speed, and to implement a zoom function on the main camera to be able to zoom in on the patient's body.

Interface element layout The doctors had a few comments about setting up the different interface elements for controlling the robot. First, they suggested to arrange the screens of the interface similar to the traditional examination, with the view of the patient to the left and the ultrasonography image in front of the doctor (compare also to Fig. 4). The second suggestion was to develop rearrangeable interface components to accommodate righthanded and left-handed users. Some of the doctors felt back pains after using the ReMeDi prototype for a while. Therefore, they suggested to include an arm rest below the haptic handle to increase the ergonomics of the interface.

General comments All doctors pointed out that they used the ReMeDi system successfully, even at the prototype stage. They were able to align the probe on the pre-defined points of the patient body, although sometimes needing longer times. The doctors also positively noticed that it was possible to rotate the ultrasonic probe on the patient's body, which is a vital part of an ultrasonography examination. Most doctors mentioned that they would need a proper training session before being able to productively use the system every day.

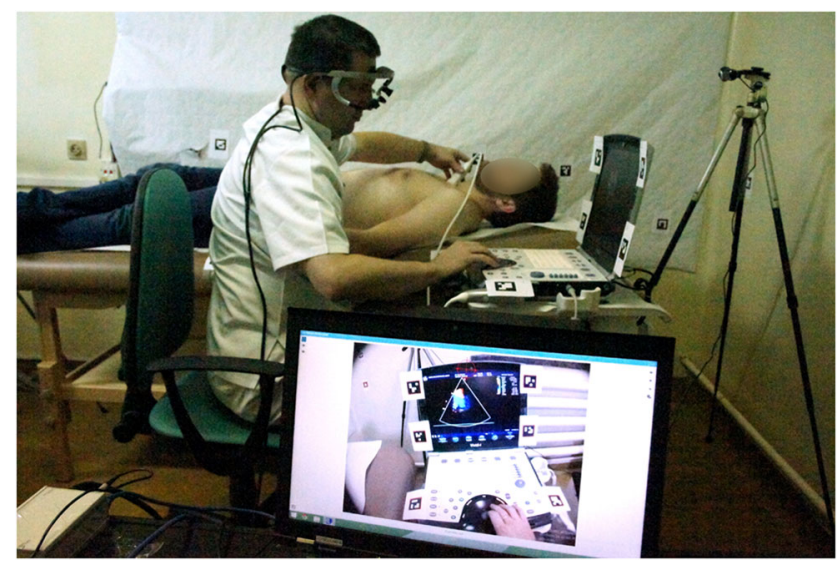

Fig. 4 Setup of eye tracking study for traditional examination. Doctor uses a mobile ultrasonography device for echocardiography. Screen in foreground shows live eye tracking preview 
In summary, the feedback and comments by the doctors were very valuable and led to changes in system design for the second prototype of the ReMeDi system. We changed the procedure to synchronise the haptic interface to the robot arm. It turned out that the problems the users had with synchronisation was due to the visualisation of a virtual probe handle shown on the patient screen, in combination with the camera angle of the camera pointing towards the patient. An improved virtual handle visualisation and optimised camera angle solved the synchronisation problem as well as the other mentioned camera angle shortcoming of the robot arm blocking the view. Additionally, we decreased the delay for robot arm control, vision system, and video conferencing system. The robot arm was completely redesigned in the second iteration of the ReMeDi system. It is less noisy and lighter than the first robot arm and drives with less force, to ensure patient safety, and its kinematics avoid driving into arm limitations. We furthermore restructured the interface elements to resemble a more traditional examination setup (see Fig. 1a). Finally, we equipped the haptic interface with an armrest to increase economic usage of the handle and covered the interface's mechanical parts and motors with a piece of cloth to avoid doctors reaching into them.

There were two comments by the doctors that we could not address in the second system prototype. Some of the doctors asked to make the system adaptable for left-hand and right-hand users. This could be implemented in future iterations of the system by rearranging the layout of the interface elements for the doctor. The doctors furthermore asked whether the alignment of the haptic interface and the ultrasonic probe could be automated. For this, the haptic interface needs to be equipped with additional motors to move the probe-shaped handle, which was outside of the project scope.

We tested the second prototype of the ReMeDi system with a similar test protocol to the first prototype evaluation, this time using a real ultrasonic probe and a real patient instead of a practice dummy. We also used eye tracking to compare this remote examination to a traditional examination. The next section describes the study setup and results for these two studies.

\section{Evaluation: eye tracking comparison studies}

In this section, we describe the final step in the UCD cycle, the evaluation of the robot together with end users for which we used two eye tracking studies. The main goal of these studies was to compare focus of attention, task execution times, and gaze fixation of traditional echocardiography examination to remote examination with the ReMeDi system. For that, we asked doctors to wear a mobile eye tracker while performing the two types of examination. We specifically chose to use eye tracking studies to compare traditional to remote examination in order to see whether the gaze patterns and fixation times that user automatically apply in both applications are similar. We first describe the method, participants, and materials of both studies (Sections 6.1 and 6.2. After that, we report and compare the results of both studies (Section 6.3). Finally, we discuss the results in Section 7.

\subsection{Eye tracking study I: traditional echocardiography examination}

The goal of the first eye tracking study we carried out was to measure the focus of attention, task execution times, and gaze fixations of doctors performing a traditional echocardiography examination.

\subsubsection{Study setup}

Figure 4 shows the setup for the eye tracking study of the traditional examination. The participating doctors performed a complete examination of real patients on the premises of Clinical Hospital No 4 Lublin, Poland. The examination consisted of an initial interview, auscultation (depending on patient condition), palpation of the stomach (depending on patient condition), echocardiographical examination with an ultrasonography device, and documentation of the diagnostic findings. For the examination, the patients first entered the examination room and were either sitting on or standing in front of an examination settee for the interview. The doctors asked the patients to lay down on the settee for palpation and echocardiography. For echocardiography, the doctors used a mobile ultrasonic device by GE. During the whole examination, the doctors wore an Ergoneers Dikablis Professional mobile eye tracker. The tracker records a scene view in HD quality and tracks the eyes with $60 \mathrm{~Hz}$ eye tracking frequency. We equipped the areas around the ultrasonic device and around the examination settee with tracking markers to enable automated analysis of the eye tracking data. The experimenters calibrated the eye tracker for each participating doctor and then left the room during the examinations, to give privacy to doctor and patient.

We informed all doctors and patients about the purpose of this study. All participants signed an informed consent form. The ethics committee of Clinical Hospital No 4 Lublin reviewed and approved this study. 


\subsubsection{Participants}

9 doctors (5 male, 4 female) and 24 patients took part in this study. The mean age of the doctors was 34.6 years $(\mathrm{SD}=4.3)$. The areas of expertise of the doctors were echocardiography, cardiology, radiology, and ultrasonography. We recruited all doctors from the hospital staff at Clinical Hospital No 4 Lublin. The doctors reported that they had been working as doctors for 8.2 years on average $(\mathrm{SD}=4.1)$. Although the doctors had expertise in different clinical areas, they were all regular users of ultrasonography systems and they all did regularly execute echocardiography examinations. The patients were regular patients of the hospital. We did not collect any personal details, such as age, gender, and health condition, to protect the privacy of the patients.

\subsubsection{Eye tracking data}

We excluded the eye tracking data of 4 examinations, because they were abdominal ultrasonography examinations, which we cannot execute with the ReMeDi robot. Furthermore, we had to exclude data from 1 echocardiography examination, because the eye tracker was not calibrated correctly. From the 19 remaining examinations, we recorded data consisting of the tracked position of the eye gaze and two synchronised video streams from the field camera of the eye tracker (1920 x 1080 pixel resolution) and from an external USB camera (640 x 480 pixel resolution). We did not record a sound source during the examinations. We defined several areas of interest (AOI) in the viewing field of the doctors. Figure 5 shows the AOIs for the patient's body and face, as well as the screen and keyboard of the ultrasonic device. Additionally, we defined an AOI to the right of the ultrasonic device on a table where the doctors placed their papers for documenting the diagnostic findings (not shown in figure). We used Ergoneers D-Lab to define the AOIs using the tracking markers visible in the field of view of the eye tracker.

\subsection{Eye tracking study II: remote echocardiography examination}

In the second eye tracking study, we measured the focus of attention, task execution times, and gaze fixations of doctors performing a remote echocardiography examination with the ReMeDi robot.

\subsubsection{Study setup}

As with the first study, we performed the second eye tracking study at the premises of Clinical Hospital No 4 Lublin. The participants were all doctors from the hospital. Since, in this study, we were interested in the doctors' perspective, the patients were healthy members of the project team. The doctors were welcomed to the study room and they were asked to give their consent to participate in the study. Next, the participants were given a short introduction
Fig. 5 Tracked areas of interest during the traditional examination

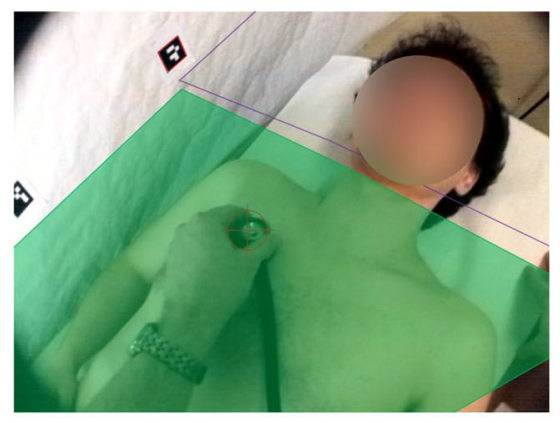

(a) Patient body.

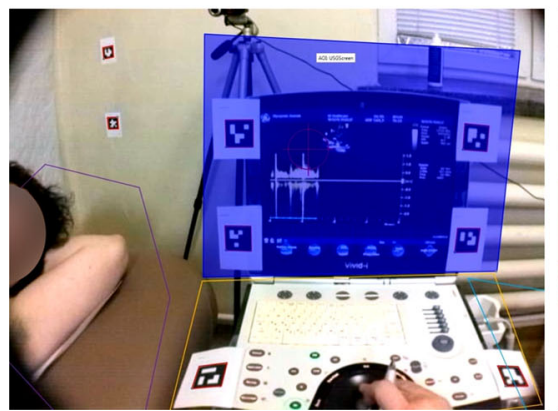

(c) USG screen.

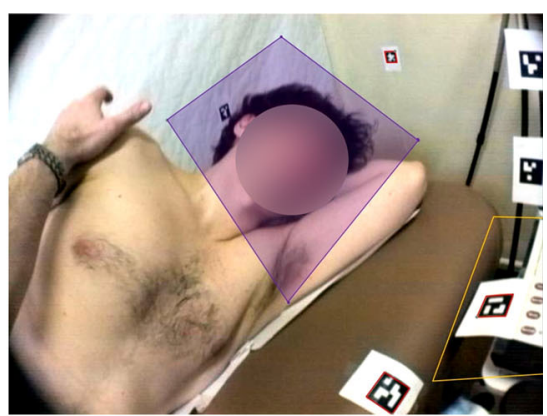

(b) Patient face

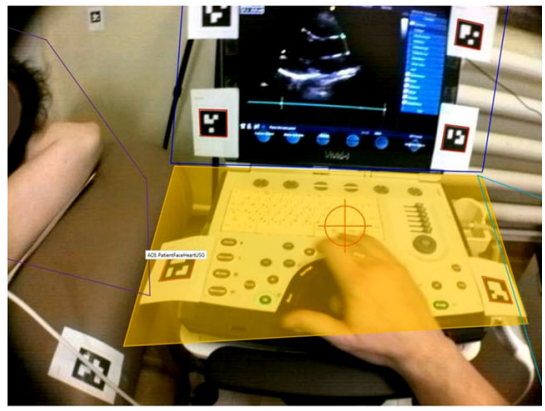

(d) USG keyboard. 


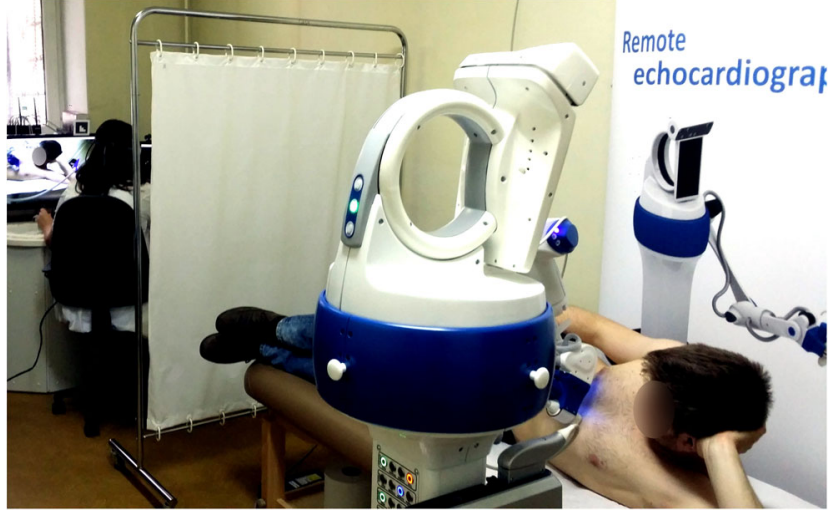

Fig. 6 Setup of eye tracking study for remote examination. Patient receives echocardiography examination remotely controlled by doctor

to the ReMeDi robot (Fig 6), in which they were shown how to operate the robot and how to place the ultrasonography probe on the patient's body using the robot. After the introduction the doctors were given as much time as they wanted to train with the robot. Only when the doctors could operate the ultrasonographic probe well with the help of the robot, the researchers calibrated the eye tracker for each participating doctor and then the echocardiographic examination began. During the examination, the doctors wore the same Ergoneers Dikablis Professional mobile eye tracker already used in the traditional examination (see Section 6.1). Similarly to the previous study, we used markers to enable automated analysis of the eye tracking data (see Fig. 7). The ethics committee of Clinical Hospital No 4 Lublin reviewed and approved the study.

Figure 8 shows the doctor's workspace. With her left hand, the doctor operated the haptic device with which she moved the ultrasonic probe (Fig. 8a). The left screen shows a live feed of the patient and the robot arm moving the probe (Fig. 8c). The screen in the middle and the keyboard in front

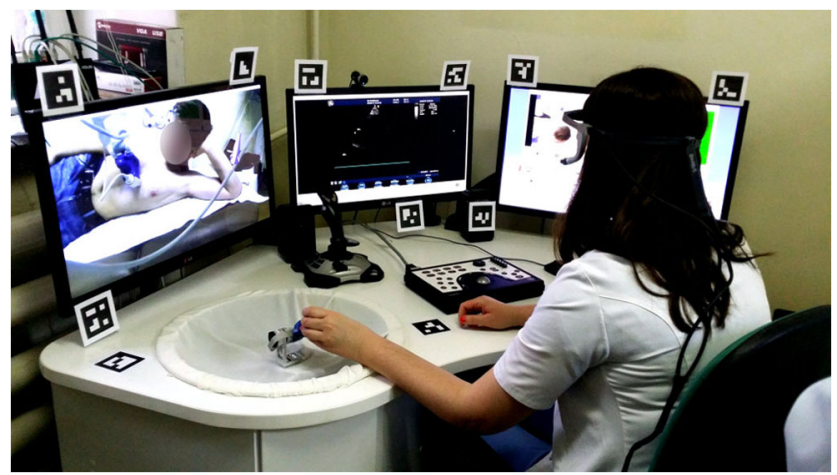

Fig. 7 Setup of eye tracking study for remote examination. Doctor controls the robot with the ReMeDi interface and wears a mobile eye tracker during the examination of it are the ultrasonography device (Fig. 8b). The screen on the right shows a three-way video conferencing system between doctor, assistant, and patient [39].

\subsubsection{Participants}

In this study, 8 doctors ( 5 male, 3 female) examined 14 patients. We recruited all doctors from the hospital staff at Clinical Hospital No 4 Lublin. As stated previously, the patients in this study were part of the ReMeDi project team, since we were only interested in the doctors' perspective. Due to hospital emergencies, 2 doctors did not have the opportunity to complete the questionnaires. In the following, we report the eye tracking results from 8 doctors and the questionnaire data from the remaining 6 doctors. Three doctors had gained previous experience with the ReMeDi system and three doctors were new to the system. Participants' age ranged from 34 to 58 years (mean = $41.00, \mathrm{SD}=8.85$ ). Their experience in echocardiography ranged from 10 to 33 years $($ mean $=15.16, \mathrm{SD}=8.93$ ). Five doctors reported to regularly perform ultrasonography examinations, while one doctor only rarely performed this kind of examination, but knew how to perform a regular examination.

\subsubsection{Eye tracking data}

We recorded data consisting of the tracked position of the eye gaze and two synchronised video streams from the field camera of the eye tracker (1920 x 1080 pixel resolution). We did not record sound during the examinations. In alignment to the eye tracking study on traditional ultrasonography examination, we defined several areas of interest (AOI) in the viewing field of the doctors. Figure 8 shows the AOIs for the USG probe handle, the USG screen and keyboard, the patient screen, and the additional screen. We used Ergoneers D-Lab to define the AOIs using the tracking markers visible in the field of view of the eye tracker.

\subsubsection{Questionnaire data}

Prior to the doctors' interaction with the ReMeDi system, we asked them to complete a short questionnaire on their demographics (age, gender, pre-experience as a cardiologist and with robots). Furthermore, we asked them to rate their affinity for technology on the Attitude Towards Technology scale (ATT) that has ten 5-point Likertscaled items [12]. At the end of the user study, we asked the cardiologists to complete the System Usability Scale [11] (ten 5-point Likert-scaled items), the Perceived Safety Scale from the Godspeed Questionnaire Series [7] (three semantic differentials), and a questionnaire assessing their trust in the ReMeDi system (23 5-point Likert-scaled 
Fig. 8 Tracked areas of interest during the remote examination

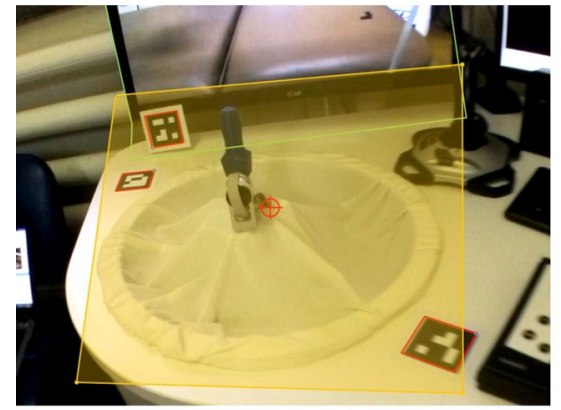

(a) USG probe handle.

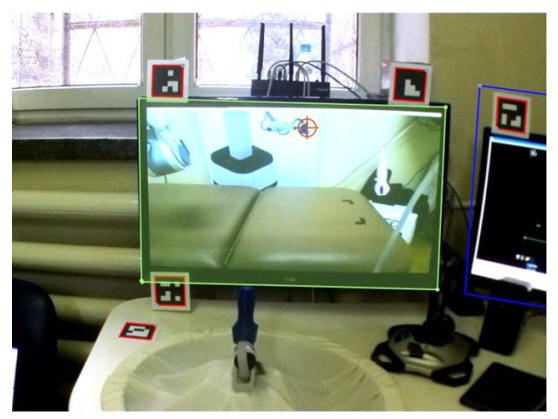

(c) Patient screen.

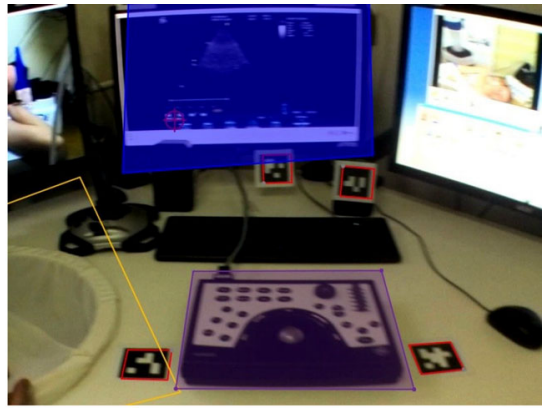

(b) USG screen and keyboard.

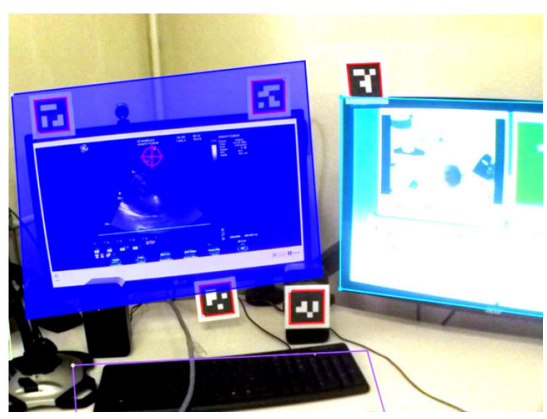

(d) USG and additional screen. items). The Trust Questionnaire was combined with subscales from [28] (functionality, reliability, helpfulness), [41] (perceived usefulness, perceived ease of use), and [25] (system trust). Finally, the doctors were asked three open questions regarding their opinion on the system.

\subsection{Results}

\subsubsection{Average fixation ratios}

Firstly, we measured the average fixation ratios for all defined AOIs compared for each study individually. Table 2 shows the average fixation ratios in percentage and their standard deviations for the traditional examination for all AOIs per examination task. To remind the reader, in the first eye tracking study, we captured data from traditional examinations that included palpation, auscultation, and echocardiography. Table 2 shows the fixation ratios for all three tasks separately.

For the palpation task, the doctors mostly looked at the patient's body ( $\sim 48.60 \%$ of the time) and face $(\sim 11.04 \%)$. The doctors also looked at the wall near the examination settee, hence there is a long time $(\sim 37.53 \%)$ in which doctors did not fixate on one of the predefined AOIs (category Other in Table 2). For the auscultation task, doctors mostly looked at the patient's body $(\sim 33.03 \%)$ and face $(\sim 7.00 \%)$. During auscultation, most doctors looked away to concentrate on the patient's heartbeat. Thus, there is a high percentage of fixations outside of the predefined AOIs $(\sim 58.60 \%)$. For the echocardiography task, doctors mostly fixated on the USG screen $(\sim 69.91 \%)$ and keyboard $(\sim 9.88 \%)$. The data also shows, that the doctors started

Table 2 Average fixation ratios on areas of interest in traditional examination tasks

\begin{tabular}{llllll}
\hline AOI & $\begin{array}{l}\text { Palpation } \\
\text { Mean }\end{array}$ & Std & $\begin{array}{l}\text { Auscultation } \\
\text { Mean }\end{array}$ & Std & $\begin{array}{l}\text { Echocardio. } \\
\text { Mean } \\
\text { Std }\end{array}$ \\
\hline USG screen & $0.49 \%$ & 0.85 & $0.18 \%$ & 0.37 & $69.91 \%$ \\
USG keyboard & $2.14 \%$ & 2.49 & $0.72 \%$ & 1.22 & $9.88 \%$ \\
Patient body & $48.60 \%$ & 27.70 & $33.03 \%$ & 21.21 & $1.76 \%$ \\
Patient face & $11.04 \%$ & 13.46 & $7.00 \%$ & 10.07 & $1.22 \%$ \\
Documentation & $0.20 \%$ & 0.35 & $0.48 \%$ & 0.98 & $4.80 \%$ \\
Other & $37.53 \%$ & 24.30 & $58.60 \%$ & 25.14 & 1.15 \\
\hline
\end{tabular}


Table 3 Average fixation ratios on areas of interest in remote examination with ReMeDi

\begin{tabular}{|c|c|c|c|c|}
\hline AOI & Mean & Min & Max & SD \\
\hline USG screen & $55.42 \%$ & $14.41 \%$ & $83.62 \%$ & 17.52 \\
\hline USG keyboard & $2.25 \%$ & $0.06 \%$ & $6.60 \%$ & 2.42 \\
\hline USG probe handle & $2.21 \%$ & $0.02 \%$ & $17.81 \%$ & 5.23 \\
\hline Patient screen & $9.87 \%$ & $0.07 \%$ & $24.97 \%$ & 9.78 \\
\hline Additional screen & $1.19 \%$ & $0.01 \%$ & $4.93 \%$ & 1.63 \\
\hline Other & $29.06 \%$ & - & - & - \\
\hline
\end{tabular}

to document their findings during the echocardiography examination $(\sim 4.80 \%$ fixation time on Documentation AOI).

In comparison to the data from the traditional examination, Table 3 gives an overview on the fixation ratios on individual AOIs for the remote examination. In this study, the doctors looked at the USG screen for $\sim 55.42 \%$ of the examination time. In $\sim 9.87 \%$ of the time they looked at the patient screen, followed by the USG keyboard $(\sim 2.25 \%)$, the USG probe handle $(\sim 2,21 \%)$, and the additional screen $(\sim 1.19 \%)$. For $\sim 29.06 \%$ of the time, the cardiologists fixated regions outside the defined AOIs (category Other in Table 3).

Upon comparing the fixation times of the cardiologists when using the ReMeDi system with them performing the examination traditionally without a robot, we notice the following. When using the ReMeDi system, the cardiologists fixated less on the USG screen $(\sim-15 \%)$ and USG keyboard $(\sim-7 \%)$, and they fixated more on the patient $(\sim+7 \%)$ and more on other areas outside our predefined AOIs $(\sim+16 \%)$.

The results show on one hand, that the focus of attention is quite similar between traditional and remote examination. Most attention lies on the USG screen, which suggests that we transferred the traditional examination needs quite well into the interaction design of the ReMeDi system. There was slightly more visual attention on the patient screen in the remote examination than on the actual patient in the traditional examination, which is due to the prior rough positioning of the robotic arm.

\subsubsection{Task execution times}

Table 4 shows the task execution times for the separate tasks of the 19 traditional echocardiography examinations (palpation, auscultation, echocardiography) from the first eye tracking study and the echocardiography of the 14 remote examinations. For the traditional examination, we have no exact data for the duration of the interviews and the documentation of findings, since the doctors were often interrupted during these tasks, e.g., by colleagues who asked questions in between patients. The data from the traditional examination shows that the doctors need relatively short times for palpation $(\sim 37.3 \mathrm{~s})$ and auscultation $(\sim 51.2 \mathrm{~s})$ in comparison to echocardiography, which takes them over 15 minutes ( $\sim 937.7 \mathrm{~s})$ on average. For the remote examination, which consisted only of the echocardiography task, doctors on average needed over 19 minutes $(\sim 1153.0$ s) to complete the task. Table 4 also shows that the doctors, who completed the task the fastest, took comparable times in traditional and remote examination (631.6s vs. 653.8s). Doctors, who needed more time took almost twice as long in the remote examination than in the traditional examination $(2165.5 \mathrm{~s}$ vs. 1272.5 s) to complete the task.

\subsubsection{Number and duration of fixations and glances}

Table 5 shows the number of fixations and glances and their durations. We included fixations and glances of both eye tracking studies for comparable AOIs in the same table. From the data we see that the doctors have a much higher

Table 4 Task execution times in seconds for subtasks in traditional and remote examination

\begin{tabular}{lllll}
\hline Task & Mean & Min & Max & Std \\
\hline Trad. Palpation & 37.321 & 10.336 & 66.782 & 18.417 \\
Trad. Auscultation & 51.160 & 15.515 & 83.511 & 20.538 \\
Trad. Echocardiography & 937.719 & 631.584 & 1272.522 & 174.769 \\
Rem. Echocardiography & 1152.971 & 653.761 & 2165.530 & 421.218 \\
\hline
\end{tabular}


Table 5 Comparison of number and duration of fixations and glances on different AOIs during traditional and remote examination

\begin{tabular}{|c|c|c|c|c|c|c|c|c|c|}
\hline \multirow[t]{2}{*}{ AOI } & \multirow[t]{2}{*}{ Study } & \multicolumn{2}{|c|}{ Number of Fixations } & \multicolumn{2}{|c|}{ Fixation Duration (s) } & \multicolumn{2}{|c|}{ Number of Glances } & \multicolumn{2}{|c|}{ Glance Duration (s) } \\
\hline & & Mean & Std & Mean & Std & Mean & Std & Mean & Std \\
\hline \multirow[t]{2}{*}{ USG Screen } & Traditional & 1224.68 & 305.64 & 0.514 & 0.086 & 393.05 & 209.56 & 1.959 & 0.670 \\
\hline & Remote & 582.79 & 192.07 & 1.151 & 0.612 & 547.71 & 224.02 & 1.378 & 0.860 \\
\hline \multirow[t]{2}{*}{ USG Keyboard } & Traditional & 566.74 & 251.18 & 0.132 & 0.035 & 259.00 & 162.69 & 0.395 & 0.174 \\
\hline & Remote & 131.00 & 111.86 & 0.140 & 0.086 & 125.86 & 112.25 & 0.172 & 0.126 \\
\hline Patient Face & Traditional & 149.79 & 112.66 & 0.130 & 0.068 & 83.74 & 70.82 & 0.482 & 0.677 \\
\hline Patient Screen & Remote & 259.62 & 317.52 & 0.532 & 0.229 & 196.64 & 303.26 & 0.731 & 0.458 \\
\hline Patient Body & Traditional & 40.67 & 29.05 & 0.114 & 0.049 & 27.33 & 22.03 & 0.221 & 0.128 \\
\hline Haptic Interface & Remote & 61.92 & 90.63 & 0.165 & 0.108 & 67.58 & 105.28 & 0.190 & 0.107 \\
\hline
\end{tabular}

number of fixations and glances on USG screen and USG keyboard during the traditional examination in comparison to the remote examination. However, the fixation duration and glance duration on the USG screen is shorter for the traditional examination than for the remote examination, but durations are comparable for the fixations and glances on the USG keyboard, regardless of examination type.

We decided to also include a comparison between the AOIs Patient face (traditional examination) and Patient screen (remote examination) as well as the AOIs Patient body (traditional examination) and Haptic interface (remote examination). The reasoning behind this decision was that, although these AOIs are not identical, they are still comparable, because the doctors in both cases fixate on the patient's face (Patient face vs. Patient screen) and the handle of the ultrasonography probe (Patient body vs. Haptic interface) respectively. For both of these comparisons Table 5 shows that the number of fixations and number of glances as well as their durations are not significantly different.

\subsubsection{Subjective results remote examination}

Finally, we analysed the subjective results from the questionnaires we used in the user study for the remote examination. The participants rated their own attitude towards technology with $3.82(\mathrm{SD}=.47)$ points on the Attitude Towards Technology Scale (ATT), a questionnaire that contains 10 items to assess the attitude of the participant towards technology [12]. The ATT Likert scale ranges from 1 (positive) to 5 (negative attitude).

On the Godspeed questionnaire, our participants rated the ReMeDi system as safe (mean $=4.11, \mathrm{SD}=.86$ ). The perceived safety subscale of the Godspeed questionnaire ranges from 1 (negative) to 5 (positive).

On the Trust questionnaire the participants rated their overall trust in the system above average (mean $=3.46$, $\mathrm{SD}=.322$ ). All subscales of the trust questionnaire were rated above average (Reliability: mean $=3.25, \mathrm{SD}=.39$;
Functionality: mean $=3.94 ; \mathrm{SD}=.14$; Perceived Ease of Use: mean $=3.21 ; \mathrm{SD}=.62 ;$ System Trust: mean $=3.45$; $\mathrm{SD}=.52$ ). The Trust questionnaire ranges from 1 (negative) to 5 (positive).

The participants rated the usability of the ReMeDi system with an average of $59.17(\mathrm{SD}=13.66)$ points on the System Usability Scale (SUS), which is a ten-item attitude Likert scale giving a global view of subjective assessments of usability [11]. SUS ranges from 0 (bad usability) to 100 (best usability) points. According to [6], our result corresponds to a usability between "acceptable" and "good".

\section{Discussion}

In this section, we first discuss the results of our comparison study and describe additional qualitative results to underpin our interpretation of the data. We then discuss the application of user-centred design methods in the medical context and our experience with applying these methods. Finally, we explain limitations of our work.

\subsection{Traditional vs. Remote examination}

The results from our two eye tracking studies show that in principle the doctors were able to successfully conduct a remote examination with the ReMeDi system. Our study participants rated the usability of the system between acceptable and good (on average 59.17 points of the 100 point System Usability Scale), which means that the usability still needs to be increased. In our opinion, there are two main reasons for the low usability of the system. On one hand, the doctors need extra time to correctly place the probe on the patient's body. This can be seen in the longer execution times for the overall examination and the fixation percentages. In the second study, the doctors looked longer at the screen showing patient and robot arm to make sure that the ultrasonography probe is placed correctly. On 
the other hand, the doctors need more training for operation of the system. All of our participants reported that they would need training in order to use the robot on an everyday basis. This is similar to other medical robotics fields. E.g., in minimal invasive surgery, doctors are now exclusively trained to execute operations with surgical robots.

The differences in number of fixations and glances and their durations between traditional and remote examination are noteworthy. The number of fixations on USG screen and keyboard are considerably higher in the traditional examination (screen: 1224.68 vs. 582.79 fixations, keyboard: 566.74 vs. 131.00 fixations), while at the same time, the mean duration of the fixations on the USG screen is considerably lower (screen: $0.514 \mathrm{~s}$ vs. $1.151 \mathrm{~s}$ ), compared to the remote examination. The higher fixation numbers can partly be explained by our experiment design. In the traditional examinations doctors usually put the ultrasonography probe on 4 positions of the patient's body to examine the heart from different angles. In the remote examinations, we asked the doctors to put the probe to only 3 positions, because one of the positions was not reachable inside the workspace of the robot arm. Figure 4 shows the doctor placing the probe on exactly the position that was not reachable by the robot arm, it is directly placed below the patients' neck above their collarbone. The longer duration of the fixations in the remote examination can partly be explained, because we did not use real patients to take part in the remote examination, which allowed the doctors to work under less time pressure. However, we still interpret the longer fixation durations in the remote examination as a sign for that our doctors did not trust the robot to show the ultrasonography image correctly. Hence, they took more time to ensure a correct diagnosis.

Finally, we analysed in more detail, whether the experience level of the doctors taking part in the remote examination study had an influence on their task success and questionnaire ratings. First of all, we have to declare the caveat that we only had 8 participants in the study, of which 5 participants were expert users, the 5 doctors that were part of the ReMeDi development team and had used the robot before, and the remaining 3 doctors were novice users, who had not used the robot before. Hence, the results of the questionnaire data needs to be handled with care. Especially the relatively high trust ratings in the system would have probably not achieved with only novice users. The different task execution times of our users (Table 4) show that the expert users needed less time than the novice users to complete the remote examination. We computed a Mann-Whitney-U test for the 6 completely filled out questionnaires ( 3 experts, 3 novices) and did not find any significant difference on any of the questionnaire scales. This could be interpreted as sign that different task execution times are a matter of personal preference and not a sign for problems with the system. This is also reflected by the fact that all doctors reported that they successfully used the ReMeDi system. However, it has to be noted that due to the low sample size, a statistical evaluation can only be an indication and not definitive prove for our interpretation.

\subsection{User-centred design for medical robotics development}

The medical domain is specific in that one has to deal with special user groups-not only doctors, but also patientswho have specific needs in safety and privacy. Hence, we experienced that the usage of UCD methods is on one hand especially useful for this domain. On the other hand, we found that some of the methods needed to be adjusted to fit to the medical context. Shah et al. [34] found in a literature review that time, money, and labour are the main barriers to user involvement in medical device technology development. Similar to this finding, we also experienced that it is best to move the robot near the doctors in order to reduce the time and labour that doctors had to invest and to increase the potential to collect valuable user feedback. We had to adapt some of the UCD methods we used to the hospital environment. Our tests were structured less rigidly as typical usability tests, especially during the task execution phase. Each doctor develops his/her own procedure when executing echocardiography examinations. Thus, we did not follow an exact task execution protocol during the usability tests. The thinking-aloud technique worked very well with doctors and did not need any adaptation. Eye tracking proved to be a useful tool for getting objective measurements to make the traditional and remote examination comparable to each other.

\subsection{Limitations}

We have to notice that the comparison between the traditional and the remote examination is not completely accurate. We did not have real patients as participants in the remote examination study (Section 6.2). We did not include real patients in this study due to safety concerns, the ReMeDi robot was not certified for clinical trials when we executed the study, and because we did not want to put the patients into the risk of getting an incorrect diagnosis. Furthermore, the traditional examination did include more steps in the examination procedure than the remote examination. In addition to echocardiography, the traditional examination also included auscultation and palpation. Both of these steps did not take the doctors long (37.3s and $51.2 \mathrm{~s}$ on average, respectively), still these additional steps have to be taken into account when comparing the execution times and fixation percentages for both examinations. 


\subsection{Future work}

In future work, we plan to introduce quicker ultrasonic probe placement by automating the alignment of virtual handle and the probe on the end effector. We also plan to further evaluate the second prototype of the ReMeDi system together with the end users.

\section{Conclusion}

Our work shows that in medical robotics development the usage of user-centred design methods can lead to a product that meets the requirements of the doctors, who are the end users of the system. We furthermore presented that some of the methods have to be adjusted to the clinical context, especially usability tests, which have to be executed in a more flexible way and near the users. Eye tracking proved to be a useful tool for comparing traditional and remote examination, although there were differences in the structure of both examinations and we were not able to execute the remote examination with real patients.

Our results are relevant for researchers, who apply usercentred design methods in the medical robotics context and for medical robot system builders. Working together with end users is a somewhat time-consuming process, which has to be executed carefully. However, it leads to a more usable medical product. We also strongly believe that our work contributes to the WHO's goal to increase access for patients in underdeveloped or sparsely populated areas to medical specialists.

Funding Information This work was supported by the project ReMeDi (Remote Medical Diagnostician, Grant No. 610902) funded by the European Commission through the 7th Framework Programme.

\section{Compliance with Ethical Standards}

Conflict of interests The authors declare that they have no conflict of interest.

Ethical approval The ethics committee of Clinical Hospital No 4 Lublin reviewed and approved this study.

Informed Consent We informed all doctors and patients about the purpose of this study. All participants signed an informed consent form.

Open Access This article is licensed under a Creative Commons Attribution 4.0 International License, which permits use, sharing, adaptation, distribution and reproduction in any medium or format, as long as you give appropriate credit to the original author(s) and the source, provide a link to the Creative Commons licence, and indicate if changes were made. The images or other third party material in this article are included in the article's Creative Commons licence, unless indicated otherwise in a credit line to the material. If material is not included in the article's Creative Commons licence and your intended use is not permitted by statutory regulation or exceeds the permitted use, you will need to obtain permission directly from the copyright holder. To view a copy of this licence, visit http://creativecommons. org/licenses/by/4.0/.

\section{References}

1. Adams SJ, Burbridge BE, Badea A, Langford L, Vergara V, Bryce R, Bustamante L, Mendez IM, Babyn PS. Initial experience using a telerobotic ultrasound system for adult abdominal sonography. Canadian Association of Radiologists Journal, 2017.

2. Arbeille P, Poisson G, Vieyres P, Ayoub J, Porcher M, Boulay JL. Echographic examination in isolated sites controlled from an expert center using a 2-d echograph guided by a teleoperated robotic arm. Ultrasound Med Biol. 2003;29(7):993-1000.

3. Arent K, Cholewiński M, Domski W, Drwièga M, Jakubiak J, Janiak M, Kreczmer B, Kurnicki A, Stańczyk B, SzczėśniakStańczyk D, et al. Selected topics in design and application of a robot for remote medical examination with the use of ultrasonography and ascultation from the perspective of the remedi project. J Autom Mob Robot Intell Syst. 2017;11(2):82-94.

4. Arent K, Jakubiak J, Drwiega M, Cholewiński M, Stollnberger G, Giuliani M, Tscheligi M, Szcześniak-Stańczyk D, Janowski M, Brzozowski W, et al. Control of mobile robot for remote medical examination: Design concepts and users' feedback from experimental studies. In: Proceedings of the 9th International Conference on Human System Interactions (HSI 2016). Portsmouth: IEEE; 2016. p. 76-82.

5. Avizzano CA, Filippeschi A, Villegas JMJ, Ruffaldi E. An optimal geometric model for clavels delta robot. In: Proceedings of the 2015 IEEE European Modelling Symposium; 2015, p. 232-237.

6. Bangor A., Kortum P., Miller J. Determining what Individual SUS Scores Mean Adding an Adjective Rating Scale. J Usability Stud. 2009;4(3):114-123.

7. Bartneck C, Kulić D, Croft E, Zoghbi S. Measurement instruments for the anthropomorphism, animacy, likeability, perceived intelligence, and perceived safety of robots. Int J Soc Robot. 2009;1(1):71-81.

8. Boman K, Olofsson M, Berggren P, Sengupta PP., Narula J. Robot-assisted remote echocardiographic examination and teleconsultation. JACC: Cardiovascular Imaging. 2014;7(8):799-803.

9. Boman K, Olofsson M, Forsberg J, Boström S. Remotecontrolled robotic arm for real-time echocardiography: the diagnostic future for patients in rural areas? Telemed e-Health. 2009;15(2):142-147.

10. Brandt G, Zimolong A, Carrat L, Merloz P, Staudte H-W, Lavallee S, Radermacher K, Rau G. Crigos: A compact robot for image-guided orthopedic surgery. IEEE Trans Inf Technol Biomed. 1999;3(4):252-260.

11. Brooke J, et al. Sus-a quick and dirty usability scale. Usability Eval Industry. 1996;189(194):4-7.

12. Edison SW, Geissler GL. Measuring attitudes towards general technology: Antecedents, hypotheses and scale development. J Target Measur Anal Market. 2003;12(2):137-156.

13. Georgescu M, Sacccomandi A, Baudron B, Arbeille PL. Remote sonography in routine clinical practice between two isolated medical centers and the university hospital using a robotic arm: A 1-year study. Telemed e-Health. 2016;22(4):276-281.

14. Shah SGS, Robinson I. User involvement in healthcare technology development and assessment: Structured literature review. Int J Health Care Qual Assur. 2006;19(6):500-515.

15. Gourdon A, Poignet P, Poisson G, Vieyres P, Marche P. A new robotic mechanism for medical application. In: Proceedings of the 
IEEE/ASME International Conference on Advanced Intelligent Mechatronics. IEEE; 1999. p. 33-38.

16. Holt R, Makower S, Jackson A, Culmer P, Levesley M, Richardson R, Cozens A, Williams MM, Bhakta B. User involvement in developing rehabilitation robotic devices: An essential requirement. In: 2007. ICORR 2007. IEEE 10th International Conference on Rehabilitation Robotics. IEEE; 2007. p. 196-204.

17. Janvier M-A, Soulez G, Allard L, Cloutier G. Validation of 3d reconstructions of a mimicked femoral artery with an ultrasound imaging robotic system. Med Phys. 2010;37(7):3868-3879.

18. Koizumi N, Warisawa S, Nagoshi M, Hashizume H, Mitsuishi M. Construction methodology for a remote ultrasound diagnostic system. IEEE Trans Robot. 2009;25(3):522-538.

19. Kwoh YS, Hou J, Jonckheere EA, Hayati S. A robot with improved absolute positioning accuracy for ct guided stereotactic brain surgery. IEEE Trans Biomed Eng. 1988;35(2):153-160.

20. Lee M, Rittenhouse M, Abdullah HA. Design issues for therapeutic robot systems Results from a survey of physiotherapists. J Intell Robot Syst. 2005;42(3):239-252.

21. Lewis C. Using the "thinking-aloud" method in cognitive interface design. Technical report, IBM TJ Watson Research Center. 1982.

22. Lu EC, Wang R, Huq R, Gardner D, Karam P, Zabjek K, Hébert D, Boger J, Mihailidis A. Development of a robotic device for upper limb stroke rehabilitation A user-centered design approach. Paladyn J Behav Robot. 2011;2(4):176-184.

23. Martin JL, Murphy E, Crowe JA, Norris BJ. Capturing user requirements in medical device development The role of ergonomics. Physiol Meas. 2006;27(8):R49.

24. Martinelli T, Bosson J-L, Bressollette L, Pelissier F, Boidard E, Troccaz J, Cinquin P. Robot-based tele-echography. J Ultrasound Med. 2007;26(11):1611-1616.

25. Master R, Jiang X, Khasawneh MT, Bowling SR, Grimes L, Gramopadhye AK, Melloy BJ. Measurement of trust over time in hybrid inspection systems. Hum Fact Ergon Manuf Serv Indust. 2005;15(2):177-196.

26. Masuda K, Takachi Y, Urayama Y, Yoshinaga T. Development of support system to handle ultrasound probe by coordinated motion with medical robot. In: Proceedings of the Annual International Conference of the IEEE Engineering in Medicine and Biology Society. IEEE; 2011. p. 4519-4522.

27. Mathiassen K, Fjellin JE, Glette K, Hol PK, Elle OJ. An ultrasound robotic system using the commercial robot ur5. Front Robot AI. 2016;3:1.

28. Harrison Mcknight D., Carter M, Thatcher JB, Clay PF. Trust in a specific technology: An investigation of its components and measures. ACM Trans Manag Inf Syst. 2011;2(2):12.

29. Norman DA, Draper SW. User centered system design: New perspectives on human-computer interaction. Boca Raton: CRC Press; 1986.

30. World Health Organization. Global strategy on human resources for health: Workforce 2030. Technical report, World Health Organization. 2016.
31. Priester AM, Natarajan S, Culjat MO. Robotic ultrasound systems in medicine. IEEE Trans Ultrason Ferroelectr Frequency Control. 2013;60(3):507-523.

32. Salcudean S, Bell G, Bachmann S, Zhu W, Abolmaesumi P, Lawrence P. Robot-assisted diagnostic ultrasound-design and feasibility experiments. In: Proceedings of Medical Image Computing and Computer-Assisted Intervention. Springer; 1999. p. 1062-1071.

33. Scheffler R, Cometto G, Tulenko K, Bruckner T, Liu J, Keuffel EL, Preker A, Stilwell B, Brasileiro J, Campbell J. Health workforce requirements for universal health coverage and the sustainable development goals - background paper $n .1$ to the who global strategy on human resources for health: Workforce 2030. Human Resources for Health Observer; 2016. p. 17.

34. Shah SGS, Robinson I. Benefits of and barriers to involving users in medical device technology development and evaluation. Int $\mathrm{J}$ Technol Assess Health Care. 2007;23(1):131-137.

35. Shaw B. Innovation and new product development in the UK medical equipment industry. Int J Technol Manag. 1998;15(35):433-445.

36. Smith-Guerin N, Al Bassit L, Poisson G, Delgorge C, Arbeille Ph, Vieyres P. Clinical validation of a mobile patientexpert tele-echography system using isdn lines. In: Proceedings of the 4th International IEEE EMBS Special Topic Conference on Information Technology Applications in Biomedicine. IEEE; 2003. p. 23-26.

37. Stańczyk B, Kurnicki A, Arent K. Logical architecture of medical telediagnostic robotic system. In 2016 21st International Conference on Methods and Models in Automation and Robotics (MMAR). IEEE; 2016. p. 200-205.

38. Stollnberger G, Christiane M, Giuliani M, Stadler S, Tscheligi M, Szczesniak-Stanczyk D, Stanczyk B. User requirements for a medical robotic system: Enabling doctors to remotely conduct ultrasonography and physical examination. In: Proceedings of the IEEE International Symposium on Robot and Human Interactive Communication (RO-MAN 2016). New York: IEEE. 2016.

39. Stollnberger G, Giuliani M, Mirnig N, Tscheligi M, Arent K, Bogdan K, Grzeszczak F, Szczesniak-Stanczyk D, Radoslaw Z, Wysokinski A. Designing user interfaces for different user groups: A three-way teleconference system for doctors, patients and assistants using a remote medical robot. In: Proceedings of the IEEE International Symposium on Robot and Human Interactive Communication (RO-MAN2016). New York: IEEE. 2016.

40. Taylor RH, Stoianovici D. Medical robotics in computerintegrated surgery. IEEE Trans Robot Autom. 2003;19(5):765781.

41. Venkatesh V, Davis FD. A theoretical extension of the technology acceptance model Four longitudinal field studies. Manag Sci. 2000;46(2):186-204.

42. Virzi RA. Refining the test phase of usability evaluation. How Many Subject Enough? Hum Fact. 1992;34(4):457-468.

Publisher's note Springer Nature remains neutral with regard to jurisdictional claims in published maps and institutional affiliations. 


\section{Affiliations}

Manuel Giuliani $^{1}$ (D) . Dorota Szczęśniak-Stańczyk ${ }^{2} \cdot$ Nicole Mirnig $^{3} \cdot$ Gerald Stollnberger $^{3} \cdot$ Malgorzata Szyszko $^{4}$. Bartlomiej Stańczyk ${ }^{4}$ - Manfred Tscheligi ${ }^{3}$

Dorota Szczęśniak-Stańnczyk

dorotaszczesniakstanczyk@umlub.pl

Nicole Mirnig

nicole.mirnig@sbg.ac.at

Gerald Stollnberger

gerald.stollnberger@sbg.ac.at

Ma lgorzata Szyszko

m.szyszko@accrea.com

Bartlomiej Stańczyk

b.stanczyk@accrea.com

Manfred Tscheligi

manfred.tscheligi@sbg.ac.at

1 Bristol Robotics Laboratory, University of the West of England,

Bristol, UK

2 Department of Cardiology, Medical University of Lublin,

Lublin, Poland

3 Center for Human-Computer Interaction, University of Salzburg,

Salzburg, Austria

4 Accrea Engineering, Lublin, Poland 